\title{
The Distribution of Anaerobic Iron \\ Oxidation in the Eastern Tropical North Pacific Oxygen Deficient Zone
}

KENNETH M. BOLSTER ${ }^{1}$, JACQUELYN A. NEIBAUER ${ }^{2}$, RICHARD G. KEIL ${ }^{2}$, JAMES W. MOFFETT ${ }^{1,3}$

${ }^{1}$ Department of Earth Sciences, University of Southern California, Los Angeles, CA, USA, kbolster@usc.edu

${ }^{2}$ School of Oceanography, University of Washington, Seattle, WA, USA

${ }^{3}$ Department of Biological Sciences, University of Southern California, Los Angeles, CA, USA

Oxygen deficient zones (ODZs) are regions of the ocean in which the water column is partially anoxic. These regions are enriched in dissolved iron(II), and are known to be an important pathway for the transport of iron away from continental shelves to open ocean basins. As a part of this "shelf to basin shuttle", iron(II) is oxidized in the water column by microbes using nitrate or nitrite as an electron acceptor in order to form oxyhydroxide minerals. Evidence for this process has been observed by different research groups in multiple ODZs, but direct measurements of anaerobic iron oxidation are difficult since most incubation techniques are not optimized to be simultaneously anaerobic and trace metal clean. We have measured those rates throughout the water column at several sites within the Eastern Tropical North Pacific ODZ, and used those observations in order to fit a model for iron transport within the ODZ. The resulting model allows us to estimate the rate of iron input to the ocean basins in these regions, and its potential impact on ocean biogeochemistry. 